\title{
Erratum: Discrete Time Crystals: Rigidity, Criticality, and Realizations [Phys. Rev. Lett. 118, 030401 (2017)]
}

\author{
N. Y. Yao, A. C. Potter, I.-D. Potirniche, and A. Vishwanath \\ (Received 14 April 2017; published 27 June 2017)
}

DOI: 10.1103/PhysRevLett.118.269901

A few errors are corrected as follows.

(i) Equation (1) omits the presence of disorder in the coupling strength, which was included in the simulations leading to the data shown in Figs. 1-3. The equation and text beneath should read

$$
H_{f}(t)= \begin{cases}H_{1} \equiv(g-\epsilon) \sum_{i} \sigma_{i}^{x}, & 0<t<T_{1} \\ H_{2} \equiv \sum_{i} J_{i}^{z} \sigma_{i}^{z} \sigma_{i+1}^{z}+B_{i}^{z} \sigma_{i}^{z}, & T_{1}<t<T,\end{cases}
$$

where $\vec{\sigma}$ represents Pauli operators, $J_{i}^{z} \in\left[J_{z}-\delta J_{z}, J_{z}+\delta J_{z}\right]$, with $\delta J_{z}=0.2 J_{z}$, and where $B_{i}^{z} \in[0, W]$ is a random longitudinal field.

We note that this $\delta J_{z}$ disorder was originally added to emulate the spatial inhomogeneity of the coupling matrix for trapped ion experiments and to remove an additional pathology of the $\delta J_{z}=0$ model in the $\epsilon=0$ limit where the end-toend mutual information exceeds $\log 2$.

Additionally, it has been noted that such coupling strength disorder is essential for stabilizing time crystalline order in the model [1].

(ii) There is a factor of 2 missing in the description of the inset of Fig. 2(a). The interaction strengths are sampled from a distribution: $J_{i}^{z} \in\left[0,2 J_{z}\right]$.

(iii) The manuscript was published online on 18 January 2017 with a production error in the text on page 1 . On page 1 , the third sentence of the second paragraph in the left-hand column should read, "However, this proof leaves the door open to TTSB in an intrinsically out-of-equilibrium setting, and pioneering recent work $[10,11]$ has demonstrated that quantum systems subject to periodic driving can indeed exhibit...."

The numerical results presented were computed with the above formulas and parameters, and the conclusions of the Letter are unmodified.

[1] We thank V. Khemani, C. von Keyserlingk, and S. Sondhi for pointing this out to us. 